\title{
Algebraic Analysis of a 3-RUU Parallel Manipulator
}

\author{
Thomas Stigger ${ }^{1}$, Abhilash Nayak ${ }^{2}$, Stéphane Caro ${ }^{3}$, Philippe Wenger ${ }^{3}$, \\ Martin Pfurner ${ }^{1}$, and Manfred Husty ${ }^{1}$ \\ 1 Unit Geometry and CAD, University of Innsbruck, 6020 Innsbruck, Austria, \\ \{thomas.stigger, martin.pfurner, manfred.husty\}@uibk.ac.at \\ 2 École Centrale de Nantes, Laboratoire des Sciences du Numérique de Nantes (LS2N), 1 rue \\ de la Noë, UMR CNRS 6004, 44321 Nantes, France, \\ abhilash.nayakels2n.fr \\ 3 CNRS, Laboratoire des Sciences du Numérique de Nantes (LS2N), École Centrale de Nantes, \\ 1 rue de la Noë, UMR CNRS 6004, 44321 Nantes, France, \\ \{stephane.caro, philippe.wenger\}@ls2n.fr
}

\begin{abstract}
Constraint equations of a parallel manipulator can be used to analyze their kinematic behaviour. This paper deals with the determination of the algebraic constraint equations of a 3-RUU parallel manipulator with two approaches. The first one is based on the manipulator geometry and the second one uses the Linear Implicitization Algorithm. The obtained constraint equations through the former approach can be given a geometrical interpretation while the latter approach is less prone to missing physical constraints. Both the ideals of constraint polynomials should lead to the same variety. Furthermore, the simplest set of equations is chosen to solve the direct kinematics problem. For the manipulator under study, it turns out that its direct kinematics problem leads to a factorisable univariate polynomial and a translational operation mode appears.
\end{abstract}

Keywords: 3-RUU, kinematic analysis, direct kinematics, algebraic geometry

\section{Introduction}

For theoretical and practical purposes, the kinematic analysis of a parallel manipulator (PM) is essential to understand its motion behavior. Kinematic constraints can be transformed via Study's kinematic mapping into algebraic constraint equations. Every configuration of the PM is thereby mapped to a point in a projective space, $\mathbb{P}^{7}[3,4]$. Consequently, well developed concepts of algebraic geometry [1] can be used to interpret the algebraic constraint equations to obtain necessary information about the PM.

In that vein, many PMs were investigated using algebraic geometry concepts. Resultant methods were adopted to solve the direct kinematics of Stewart-Gough platforms [2]. A complete kinematic analysis including the characterization of operation modes, solutions to direct kinematics and determination of singular poses was performed for the 3-RPS PM [9, 10], the 3-RPS cube PM [7] and 3-PRS PMs with different arrangements of prismatic joints [5]. In the foregoing papers, the prismatic joints were considered to be actuated, which makes the analysis inherently algebraic. A more challenging kinematic analysis of an over-constrained 4-RUU PM with square base and moving platform was accomplished by decomposing it into two 2-RUU PMs [6]. The 
constraint equations of a 3-RUU PM are derived in this paper and its direct kinematics problem is solved. Nevertheless, a complete characterization of the manipulator operation modes has not been obtained yet.

The paper is organized as follows: Section 2 describes the manipulator architecture. Section 3 deals with the derivation of algebraic constraint equations with two approaches and their comparison. Section 4 presents the solutions to direct kinematics for arbitrary design parameters and hints the recognition of a translational operation mode.

\section{Manipulator Architecture}

The 3-RUU PM is shown in Figure 1. Each limb consists of a revolute joint and two universal joints mounted in series with the first revolute joint as the active joint. The moving platform and the fixed base form equilateral triangles with vertices $C_{i}$ and $A_{i}$, respectively, $i=1,2,3$. The unit vectors of the revolute joint axes within the $i$-th limb are denoted as $\mathbf{s}_{i j}, i=1,2,3 ; j=1, \ldots, 5$. $\mathbf{s}_{i 5}$ and $\mathbf{s}_{i 1}$ are tangent to the circumcircles (with centers $P$ and $O$ ) of the moving platform and the base triangles, respectively. Vectors $\mathbf{s}_{i 1}$ and $\mathbf{s}_{i 2}$ are always parallel, so are vectors $\mathbf{s}_{i 3}$ and $\mathbf{s}_{i 4}$. The origin of the fixed coordinate frame, $\mathscr{F}_{O}$ is at $O$ and the $z_{O}$-axis lies along the normal to the base plane whereas the origin of the moving coordinate frame $\mathscr{F}_{P}$ is at $P$ and the $z_{P}$-axis lies along the normal to the moving platform plane. $x_{O}$ and $x_{P}$ axes are directed along $O A_{1}$ and $P C_{1}$, respectively. $r_{0}$ and $r_{1}$ are the circumradii of base and the moving platform, respectively. $a_{1}$ and $a_{3}$ are the link lengths. $\theta_{i 1}$ is the angle of rotation of the first revolute joint about the axis represented by vector $\mathbf{s}_{i 1}$ measured from the base plane whereas $\theta_{i 2}$ is the angle of rotation of the second revolute joint about the axis represented by vector $\mathbf{s}_{i 2}$ measured from the first link.

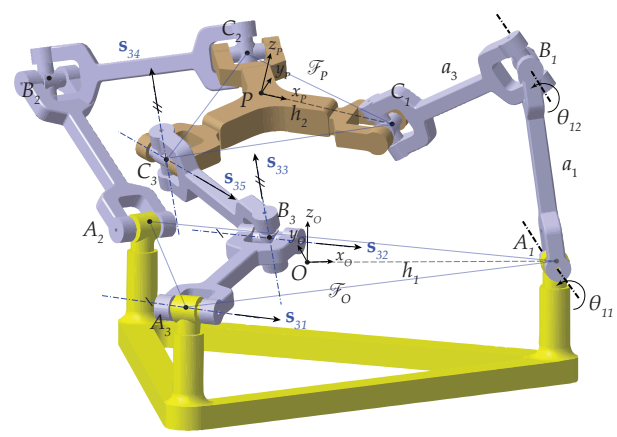

Fig. 1: The 3-RUU PM in a general configuration

\section{Constraint Equations}

The constraint equations of the 3-RUU PM are derived using a geometrical approach and the Linear Implicitization Algorithm (LIA) [11]. First, canonical constraint 


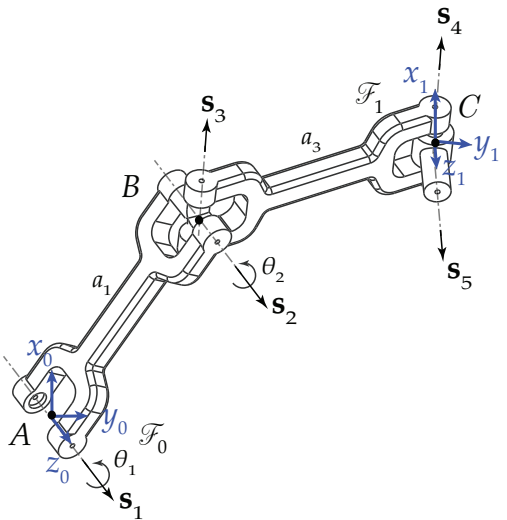

Fig. 2: A RUU limb

equations for a limb of the PM are derived by attaching fixed and moving coordinate frames to the two extreme joints of a RUU limb as shown in Fig. 2. Each U-joint is characterized by two revolute joints with orthogonal and intersecting axes and Denavit-Hartenberg (DH) convention is used to parameterize each limb. $\mathscr{F}_{0}$ and $\mathscr{F}_{1}$ are the fixed and the moving coordinate frames with their corresponding $z$-axes along the first and the last revolute joint axes, respectively. Later on, general constraint equations are derived for the whole manipulator.

\subsection{Geometrical Approach}

Canonical Constraints In order to derive the geometric constraints for a RUU limb, the homo-

geneous coordinates $4^{4}$ of points $A, B, C\left(\mathbf{a}, \mathbf{b}, \mathbf{c}\right.$, respectively) and vectors $\mathbf{s}_{j}, j=1, \ldots, 5$, shown in Fig. 2 are expressed as follows:

$$
\begin{aligned}
& { }^{0} \mathbf{a}=[1,0,0,0]^{T} \quad{ }^{0} \mathbf{b}=\left[1, a_{1} \cos \left(\theta_{1}\right), a_{1} \sin \left(\theta_{1}\right), 0\right]^{T} \quad{ }^{1} \mathbf{c}=[1,0,0,0]^{T} \\
& { }^{0} \mathbf{s}_{1}=[0,0,0,1]^{T} \quad{ }^{0} \mathbf{s}_{2}=[0,0,0,1]^{T} \quad{ }^{0} \mathbf{S}_{3}=\left[0, \cos \left(\theta_{1}+\theta_{2}\right), \sin \left(\theta_{1}+\theta_{2}\right), 0\right]^{T} \\
& { }^{0} \mathbf{s}_{4}=\left[0, \cos \left(\theta_{1}+\theta_{2}\right), \sin \left(\theta_{1}+\theta_{2}\right), 0\right]^{T} \quad{ }^{1} \mathbf{S}_{5}=[0,0,0,1]^{T}
\end{aligned}
$$

where $\theta_{1}$ and $\theta_{2}$ are the angles of rotation of the first and the second revolute joints.

Study's kinematic mapping is used to express the vectors $\mathbf{c}$ and $\mathbf{s}_{5}$ in the fixed coordinate frame $\mathscr{F}_{0}$, using the transformation matrix ${ }^{0} \mathbf{T}_{1}$ consisting of Study parameters $x_{i}$ and $y_{i}, i=0,1,2,3$ :

$$
\text { where }{ }^{0} \mathbf{T}_{1}=\frac{1}{\Delta}\left[\begin{array}{cccc}
\Delta & { }^{0} \mathbf{c}={ }^{0} \mathbf{T}_{1}{ }^{1} \mathbf{c} & \text { and } & { }^{0} \mathbf{s}_{5}={ }^{0} \mathbf{T}_{1}{ }^{1} \mathbf{s}_{5}, \\
d_{1} x_{0}{ }^{2}+x_{1}{ }^{2}-x_{2}{ }^{2}-x_{3}{ }^{2} & -2 x_{0} x_{3}+2 x_{1} x_{2} & 2 x_{0} x_{2}+2 x_{1} x_{3} \\
d_{2} & 2 x_{0} x_{3}+2 x_{1} x_{2} & x_{0}{ }^{2}-x_{1}{ }^{2}+x_{2}{ }^{2}-x_{3}{ }^{2} & -2 x_{0} x_{1}+2 x_{2} x_{3} \\
d_{3} & -2 x_{0} x_{2}+2 x_{1} x_{3} & 2 x_{0} x_{1}+2 x_{2} x_{3} & x_{0}{ }^{2}-x_{1}{ }^{2}-x_{2}{ }^{2}+x_{3}{ }^{2}
\end{array}\right]
$$

with $\Delta=x_{0}^{2}+x_{1}^{2}+x_{2}^{2}+x_{3}^{2} \neq 0$ and $d_{1}=-2 x_{0} y_{1}+2 x_{1} y_{0}-2 x_{2} y_{3}+2 x_{3} y_{2}, d_{2}=$ $-2 x_{0} y_{2}+2 x_{1} y_{3}+2 x_{2} y_{0}-2 x_{3} y_{1}, d_{3}=-2 x_{0} y_{3}-2 x_{1} y_{2}+2 x_{2} y_{1}+2 x_{3} y_{0}$. All vectors are now expressed in the base coordinate frame $\mathscr{F}_{0}$ and hence the geometric constraints can be derived. The following constraints are already satisfied:

1. The first and the second revolute joint axes are parallel: $\mathbf{s}_{1}=\mathbf{s}_{2}$

2. Third and fourth revolute joint axes are parallel: $\mathbf{s}_{3}=\mathbf{s}_{4}$

3. $\overrightarrow{A B}$ is perpendicular to the first and the second revolute joint axes: $(\mathbf{b}-\mathbf{a})^{T} \mathbf{s}_{1}=0$

\footnotetext{
${ }^{4}$ left superscript $k$ denotes the vector expressed in coordinate frame $\mathscr{F}_{k}, k \in\{0,1\}$
} 
4. The second revolute joint axis is perpendicular to the third revolute joint axis: $\mathbf{s}_{2}^{T} \mathbf{s}_{3}=0$

5. Length of the link $A B$ is $a_{1}:\|\mathbf{b}-\mathbf{a}\|_{2}=a_{1}$

The remaining geometric constraints are derived as algebraic equation 5 . The second revolute joint axis, the fifth revolute joint axis and link $B C$ lie in the same plane. In other words, the scalar triple product of the corresponding vectors is null:

$$
g_{1}:(\mathbf{b}-\mathbf{c})^{T}\left(\mathbf{s}_{2} \times \mathbf{s}_{5}\right)=0
$$

Vector $\overrightarrow{B C}$ is perpendicular to the third and the fourth revolute joint axes:

$$
g_{2}:(\mathbf{b}-\mathbf{c})^{T} \mathbf{S}_{4}=0
$$

The fourth and the fifth revolute joint axes are perpendicular:

$$
g_{3}: \mathbf{s}_{4}^{T} \mathbf{s}_{5}=0
$$

Length of the link $B C$ is $a_{3}$ :

$$
g_{4}:\|\mathbf{b}-\mathbf{c}\|-a_{3}=0
$$

Furthermore, Study's quadric equation $\mathscr{S}: x_{0} y_{0}+x_{1} y_{1}+x_{2} y_{2}+x_{3} y_{3}=0$ must be taken into account. The five geometric relations $g_{1}, g_{2}, g_{3}, g_{4}, \mathscr{S}$ describe the RUU limbs of the PM under study. As a matter of fact, when the first revolute joint is actuated, each limb has four DoF and it should be possible to describe it by only two constraint equations. Eqs. (4) and (5) contain the passive joint variable $v_{2}$ along with the active joint variable $v_{1}$. Eliminating $v_{2}$ from $g_{2}$ and $g_{3}$ results in an equation that amounts to $g_{1}$. Therefore, the two constraint equations in addition to the Study quadric describing a RUU limb are $g_{1}$ and $g_{4}$, namely Eqs. (3) and (6). The polynomials $g_{1}, g_{4}$ and $\mathscr{S}$ define an ideal, which is a subset of all polynomials in the Study parameters:

$$
\mathscr{I}_{1}=\left\langle g_{1}, g_{4}, \mathscr{S}\right\rangle \subseteq k\left[x_{0}, x_{1}, x_{2}, x_{3}, y_{0}, y_{1}, y_{2}, y_{3}\right] .
$$

Explicitly these polynomials take the form:

$$
\begin{aligned}
g_{1}:= & \left(\left(x_{0} x_{1}-x_{2} x_{3}\right)\left(v_{1}^{2}-1\right)+\left(-2 x_{0} x_{2}-2 x_{1} x_{3}\right) v_{1}\right)\left(x_{0}^{2}+x_{1}^{2}+x_{2}^{2}+x_{3}^{2}\right) a_{1} \\
& -2\left(\left(x_{0}^{2}+x_{3}^{2}\right)\left(x_{1} y_{1}+x_{2} y_{2}\right)+2\left(x_{1}^{2}+x_{2}^{2}\right)\left(x_{0} y_{0}+x_{3} y_{3}\right)\right)\left(v_{1}^{2}-1\right)=0, \\
g_{4}:= & -\left(x_{0}^{2}+x_{1}{ }^{2}+x_{2}{ }^{2}+x_{3}{ }^{2}\right)\left(v_{1}{ }^{2}+1\right) a_{1}{ }^{2}+\left(4\left(y_{1} x_{0}-y_{0} x_{1}+y_{3} x_{2}-y_{2} x_{3}\right) v_{1}{ }^{2}\right. \\
& \left.+8\left(-x_{0} y_{2}+x_{1} y_{3}+x_{2} y_{0}-x_{3} y_{1}\right) v_{1}+4\left(y_{2} x_{3}-y_{3} x_{2}-y_{1} x_{0}+y_{0} x_{1}\right)\right) a_{1} \\
& +\left(\left(x_{0}{ }^{2}+x_{1}{ }^{2}+x_{2}{ }^{2}+x_{3}{ }^{2}\right) a_{3}{ }^{2}-4\left(y_{2}{ }^{2}+y_{3}{ }^{2}+y_{0}{ }^{2}+y_{1}{ }^{2}\right)\right)\left(v_{1}{ }^{2}+1\right)=0 .
\end{aligned}
$$

\footnotetext{
${ }^{5}$ cosine and sine of angles are substituted by tangent half-angles to render the equations algebraic; $\cos \left(\theta_{i}\right)=\frac{1-v_{i}^{2}}{1+v_{i}^{2}} \quad \sin \left(\theta_{i}\right)=\frac{2 v_{i}}{1+v_{i}^{2}}$ where $v_{i}=\tan \left(\theta_{i} / 2\right), i=1,2$
} 
General Constraints $g_{1}$ and $g_{4}$ are the constraint equations of an RUU limb with specially adapted coordinate systems. To assemble the PM one has to transform these equations so that the limbs get into the positions of Fig 1. It is well known [8] that the necessary transformations are linear in the image space coordinates. Due to lack of space these transformations are only shown for the derivation of the constraint equations using the LIA in $\operatorname{Sec} 3.2$ (Eq 14). One ends with six constraint equations $g_{i 1}, g_{i 4}, i=$ $1,2,3$ which form together with $\mathscr{S}=0$ and the normalization condition $\mathscr{N}: x_{0}^{2}+x_{1}^{2}+$ $x_{2}^{2}+x_{3}^{2}-1=0$ an ideal

$$
\mathscr{I}=\left\langle g_{11}, g_{14}, g_{21}, g_{24}, g_{31}, g_{34}, \mathscr{S}, \mathscr{N}\right\rangle \subseteq k\left[x_{0}, x_{1}, x_{2}, x_{3}, y_{0}, y_{1}, y_{2}, y_{3}\right]
$$

\subsection{Linear Implicitization Algorithm}

Canonical Constraints The canonical pose of a RUU limb is chosen such that the rotation axes coincide with the $\mathrm{z}$-axes and the common normals of these axes are in the directions of the $\mathrm{x}$-axes of the coordinate systems in order to derive the canonical constraint equations using LIA [11]. To describe the RUU kinematic chain using the usual Denavit-Hartenberg $(\mathrm{DH})$ parameters, the following $4 \times 4$ matrices are defined: $\mathbf{T}=\mathbf{M}_{\mathbf{i}} \cdot \mathbf{G}_{\mathbf{i}}, i=1, \ldots, 5$, where the $\mathbf{M}_{\mathbf{i}}$-matrices describe a rotation about the $z$-axis with $u_{i}$ as the rotation angle. The $\mathbf{G}_{\mathbf{i}}$-matrices describe the transformation of one joint coordinate system to the next.

$$
\mathbf{M}_{\mathbf{i}}=\left[\begin{array}{cccc}
1 & 0 & 0 & 0 \\
0 \cos \left(u_{i}\right) & -\sin \left(u_{i}\right) & 0 \\
0 & \sin \left(u_{i}\right) & \cos \left(u_{i}\right) & 0 \\
0 & 0 & 0 & 1
\end{array}\right], \quad \mathbf{G}_{\mathbf{i}}=\left[\begin{array}{cccc}
1 & 0 & 0 & 0 \\
a_{i} & 1 & 0 & 0 \\
0 & 0 & \cos \left(\alpha_{i}\right) & -\sin \left(\alpha_{i}\right) \\
d_{i} & 0 & \sin \left(\alpha_{i}\right) & \cos \left(\alpha_{i}\right)
\end{array}\right] .
$$

The parameters in $\mathbf{G}_{\mathbf{i}}$ are DH parameters encoding the distance along $x$-axis $a_{i}$, the offset along $z$-axis $d_{i}$ and the twist angle between the axes $\alpha_{i}$. The DH parameters for the RUU limb are $\alpha_{2}=\frac{\pi}{2}, \alpha_{4}=-\frac{\pi}{2}, d_{1}=a_{2}=d_{2}=d_{3}=a_{4}=d_{4}=\alpha_{1}=\alpha_{3}=0$. Computing the Study-Parameters based on the transformation matrix $\mathbf{T}$ yields the parametric representation of the limb [4]. Applying LIA yields the following quadratic canonical constraint equations $\mathscr{S}, f_{1}$ and $f_{2}$ :

$$
\mathscr{J}=\left\langle f_{1}, f_{2}, \mathscr{S}\right\rangle \subseteq k\left[x_{0}, x_{1}, x_{2}, x_{3}, y_{0}, y_{1}, y_{2}, y_{3}\right],
$$

where

$$
\begin{aligned}
f_{1}:= & \left(\left(x_{0} x_{1}-x_{2} x_{3}\right)\left(v_{1}^{2}-1\right)-\left(2 x_{0} x_{2}+2 x_{1} x_{3}\right) v_{1}\right) a_{1}+2\left(v_{1}^{2}+1\right)\left(x_{0} y_{0}+x_{3} y_{3}\right)=0 \\
f_{2}:= & -\left(x_{0}^{2}+x_{1}^{2}+x_{2}^{2}+x_{3}^{2}\right)\left(v_{1}^{2}+1\right) a_{1}^{2}+\left(4\left(y_{1} x_{0}-y_{0} x_{1}+y_{3} x_{2}-y_{2} x_{3}\right) v_{1}^{2}\right. \\
& \left.+8\left(-x_{0} y_{2}+x_{1} y_{3}+x_{2} y_{0}-x_{3} y_{1}\right) v_{1}+4\left(y_{2} x_{3}-y_{3} x_{2}-y_{1} x_{0}+y_{0} x_{1}\right)\right) a_{1} \\
& +\left(\left(x_{0}^{2}+x_{1}^{2}+x_{2}^{2}+x_{3}^{2}\right) a_{3}^{2}-4\left(y_{2}^{2}+y_{3}^{2}+y_{0}^{2}+y_{1}^{2}\right)\right)\left(v_{1}^{2}+1\right)=0
\end{aligned}
$$

General Constraints To obtain the constraint equations of the whole mechanism from the canonical constraint equations, coordinate transformations are applied in the base and moving platform. To facilitate the comparison of the constraint equations derived by two different approaches, the coordinate transformations should be consistent with 
the global frames $\mathscr{F}_{O}$ and $\mathscr{F}_{P}$ as shown in Fig. 1. The necessary transformations can be done directly in the image space $\mathbb{P}^{7}[8]$ by the mapping

$$
\left[\begin{array}{c}
x_{0} \\
x_{1} \\
x_{2} \\
x_{3} \\
y_{0} \\
y_{1} \\
y_{2} \\
y_{3}
\end{array}\right] \mapsto\left[\begin{array}{c}
2\left(v_{0}^{2}+1\right) x_{0} \\
-2 v_{0}^{2} x_{1}+4 v_{0} x_{2}+2 x_{1} \\
2\left(v_{0}^{2}+1\right) x_{3} \\
2 v_{0}^{2} x_{2}+4 v_{0} x_{1}-2 x_{2} \\
\left(\left(r_{0}-r_{1}\right) x_{1}+2 y_{0}\right) v_{0}^{2}-2 x_{2}\left(r_{0}-r_{1}\right) v_{0}+\left(-r_{0}+r_{1}\right) x_{1}+2 y_{0} \\
\left(\left(r_{0}-r_{1}\right) x_{0}-2 y_{1}\right) v_{0}^{2}+4 v_{0} y_{2}+\left(r_{0}-r_{1}\right) x_{0}+2 y_{1} \\
\left(\left(-r_{0}-r_{1}\right) x_{2}+2 y_{3}\right) v_{0}^{2}-2\left(r_{0}+r_{1}\right) x_{1} v_{0}+\left(r_{0}+r_{1}\right) x_{2}+2 y_{3} \\
\left(\left(r_{0}+r_{1}\right) x_{3}+2 y_{2}\right) v_{0}^{2}+4 v_{0} y_{1}+\left(r_{0}+r_{1}\right) x_{3}-2 y_{2}
\end{array}\right] \text {, }
$$

where $v_{0}=\tan \left(\gamma_{i}\right), i=1,2,3, \gamma_{1}=0, \gamma_{2}=\frac{2 \pi}{3}$ and $\gamma_{3}=\frac{4 \pi}{3}$. The general constraint equations are obtained by transforming the $f_{i}$ of Eq 12 with Eq 14 . The transformed equations are denoted $f_{i 1}=f_{i 2}=0, i=1,2,3$, and determine together with $\mathscr{S}=0$ and $\mathscr{N}=0$, the ideal $\mathscr{J}$ :

$$
\mathscr{J}=\left\langle f_{11}, f_{12}, f_{21}, f_{22}, f_{31}, f_{32}, \mathscr{S}, \mathscr{N}\right\rangle \subseteq k\left[x_{0}, x_{1}, x_{2}, x_{3}, y_{0}, y_{1}, y_{2}, y_{3}\right]
$$

\subsection{Ideal Comparison}

A careful observation of the ideals spanned by the canonical constraint polynomials of both approaches reveals that $g_{4}=f_{2}$ and $g_{1}=f_{1}\left(x_{0}^{2}+x_{1}^{2}+x_{2}^{2}+x_{3}^{2}\right)-2\left(x_{0}^{2}+x_{2}^{2}\right)\left(v_{1}^{2}+\right.$ 1) $\mathscr{S}$. Since $x_{0}^{2}+x_{1}^{2}+x_{2}^{2}+x_{3}^{2}$ cannot be null, these ideals are the same. Thus, it follows that the ideals $\mathscr{I}$ and $\mathscr{J}$ spanned by the constraint equations of the whole manipulator are also contained in each other: $\mathscr{I} \subseteq \mathscr{J} \subseteq \mathscr{I}$. Since $\mathscr{I}$ and $\mathscr{J}$ determine the same ideal, the variety of the constraint polynomials must be the same [1]. Therefore, the set of constraint equations derived in Section 3.2 is used for further computations as it contains only quadratic equations.

\section{Direct Kinematics: Numerical Examples}

Because of the complexity of the manipulator, it is not possible to compute the direct kinematics without using some numerical values. In the following subsections, the following arbitrary values are assigned to the design parameters of the manipulator, $a_{1}=3, a_{3}=5, r_{0}=11, r_{1}=7$.

Identical Actuated Joints Assuming the actuated joint angles are equal, $\theta_{i 1}=\frac{\pi}{2}, i=$ 1,2,3 for simplicity, the system of constraint equations in Eq. 15] yields the following real solutions and the corresponding manipulator poses are shown in Fig. 3 .
(a) $\left\{x_{0}=\frac{\sqrt{23023}}{154}, y_{3}=-\frac{3}{2} x_{0}, x_{3}=-\frac{3 \sqrt{77}}{154}, y_{0}=\frac{3}{2} x_{3}, x_{1}=x_{2}=y_{1}=y_{2}=0\right\}$,
(b) $\left\{x_{0}=\frac{\sqrt{23023}}{154}, y_{3}=-\frac{3}{2} x_{0}, x_{3}=\frac{3 \sqrt{77}}{154}, y_{0}=\frac{3}{2} x_{3}, x_{1}=x_{2}=y_{1}=y_{2}=0\right\}$, 


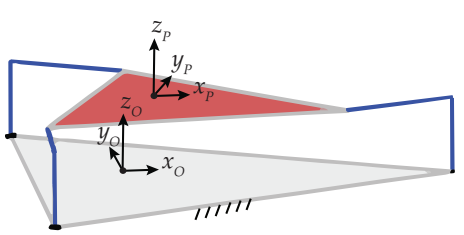

(a)

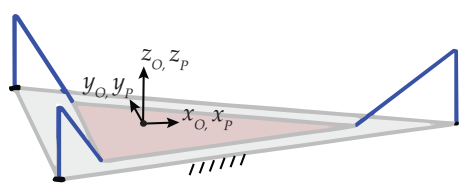

(c)

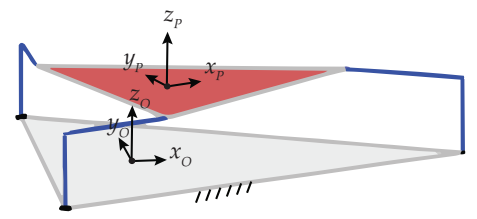

(b)

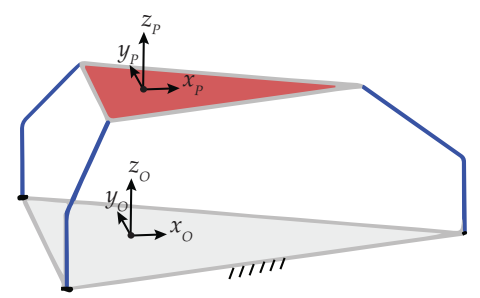

(d)

Fig. 3: A numerical example: solutions to direct kinematics corresponding to 16)

(c) $\left\{x_{0}=1, x_{1}=x_{2}=x_{3}=y_{0}=y_{1}=y_{2}=y_{3}=0\right\}$,

(d) $\left\{x_{0}=1, x_{1}=x_{2}=x_{3}=y_{0}=y_{1}=y_{2}=0, y_{3}=-3\right\}$.

Different Actuated Joints Substituting distinct arbitrary inputs, setting $x_{0}=1$ and computing a Groebner basis of the resulting polynomials with pure lexicographic ordering yields a univariate polynomial

$$
x_{3} \cdot P\left(x_{3}\right)=0, \quad \text { where } \operatorname{degree}\left(P\left(x_{3}\right)\right)=80 .
$$

Translational Operation Mode The univariate polynomial of the previous section shows that this manipulator exhibits two operation modes. The one corresponding to $x_{3}=0$ yields pure translational motions of the moving platform with the identity as the orientation. From $\mathscr{S}$ follows also $y_{0}=0$. The set of original constraint equations reduces to

$$
\begin{aligned}
& {\left[\left(3 y_{3}-y_{1}^{2}-y_{2}^{2}-y_{3}^{2}-4 y_{1}\right) t_{1}^{2}-6\left(y_{1}+2\right) t_{1}-y_{1}{ }^{2}-y_{2}{ }^{2}-y_{3}{ }^{2}-4 y_{1}-3 y_{3},\right.} \\
& -\left(2 t_{2}^{2}+3 t_{2}+2\right) y_{2} \sqrt{3}+\left(-y_{1}{ }^{2}-y_{2}{ }^{2}-y_{3}^{2}+2 y_{1}+3 y_{3}\right) t_{2}^{2}+\left(3 y_{1}-12\right) t_{2}-y_{1}{ }^{2} \\
& -y_{2}{ }^{2}-y_{3}^{2}+2 y_{1}-3 y_{3},\left(2 t_{3}^{2}+3 t_{3}+2\right) y_{2} \sqrt{3}+\left(-y_{1}{ }^{2}-y_{2}{ }^{2}-y_{3}{ }^{2}+2 y_{1}+3 y_{3}\right) t_{3}{ }^{2} \\
& \left.+\left(3 y_{1}-12\right) t_{3}-y_{1}{ }^{2}-y_{2}{ }^{2}-y_{3}{ }^{2}+2 y_{1}-3 y_{3}\right] .
\end{aligned}
$$

This system of equations yields a quadratic univariate in one of the $y_{i}$ variables, which gives a parametrization of the motion as a function of the input variables $v_{i 1}=\tan \left(\theta_{i 1} / 2\right)$, $i=1,2,3$. 


\section{Conclusion}

In this paper, the constraint equations of a 3-RUU PM were derived by two different approaches: geometrical approach, where all possible constraints were listed based on the geometry of the manipulator and through LIA in which it was enough to specify the parametric equations and the degree of the constraint equations and applying the algorithm yielded the constraints. Both approaches have benefits and disadvantages such that it is possible to miss a constraint by merely observing the manipulator geometry while it is hard to interpret the physical meaning of the equations derived through LIA. However, it turns out that the ideals spanned by the constraint polynomials with both approaches are the same. As a result, the simplest set of equations was chosen for further analysis. Due to the complexity of the mechanism, a primary decomposition of these ideals is not possible and therefore a final answer to possible operation modes can not be given. However, the factorization of the final univariate polynomial of the direct kinematics algorithm gives strong evidence that this manipulator has a translational and a general three DoF operation mode.

\section{Acknowledgements}

This work was conducted with the support of the University of Innsbruck and the support of the FWF project I 1750-N26, and also École Centrale Nantes and the French National Research Agency (ANR project Kapamat \#ANR-14-CE34-0008-01).

\section{References}

1. Cox, D.A., Little, J., O'Shea, D.: Ideals, Varieties, and Algorithms: An Introduction to Computational Algebraic Geometry and Commutative Algebra, 3rd edn. Springer (2007)

2. Husty, M.: An algorithm for solving the direct kinematics of general Stewart-Gough platforms. Mechanism and Machine Theory 31(4), 365 - 379 (1996)

3. Husty, M., Schröcker, H.P.: 21st Century Kinematics, chap. Kinematics and Algebraic geometry, pp. 85-123. Springer (2012)

4. Husty, M.L., Pfurner, M., Schröcker, H.P., Brunnthaler, K.: Algebraic methods in mechanism analysis and synthesis. Robotica 25, $661-675$ (2007)

5. Nurahmi, L., Caro, S., Wenger, P.: Operation modes and singularities of 3-PRS parallel manipulators with different arrangements of P-joints. In: DETC 2015. ASME (2015)

6. Nurahmi, L., Caro, S., Wenger, P., Schadlbauer, J., Husty, M.: Reconfiguration analysis of a 4-RUU parallel manipulator. Mechanism and Machine Theory 96, 269-289 (2016)

7. Nurahmi, L., Schadlbauer, J., Husty, M., Wenger, P., Caro, S.: Kinematic analysis of the 3-RPS Cube Parallel Manipulator. ASME Journ. of Mech. and Robotics 7(1), 1-11 (2015)

8. Pfurner, M.: Analysis of spatial serial manipulators using kinematic mapping. Doctoral thesis, University Innsbruck (2006)

9. Schadlbauer, J., Nurahmi, L., Husty, M., Wenger, P., Caro, S.: IAK: Proc. of the Intern. Conf., Lima, Peru, September 9-11, 2013, chap. Operation Modes in Lower-Mobility Parallel Manipulators, pp. 1-9. Springer International Publishing (2015)

10. Schadlbauer, J., Walter, D.R., Husty, M.L.: The 3-RPS parallel manipulator from an algebraic viewpoint. Mechanism and Machine Theory 75, 161-176 (2014)

11. Walter, D.R., Husty, M.L.: On implicitization of kinematic constraint equations. Machine Design and Research 26, 218-226 (2010) 